\title{
Effect of nutmeg extract on the white adipose tissue (WAT) browning process of aging rats
}

\author{
Yuni Susanti Pratiwi ${ }^{\mathrm{a}, \mathrm{b}}$, Melisa Siannoto ${ }^{\mathrm{c}}$, Hanna Goenawan ${ }^{\mathrm{a}, \mathrm{b}}$, Nova Sylviana ${ }^{\mathrm{a}, \mathrm{b}}$, \\ Vita Murniati Tarawan ${ }^{\text {a }}$, Titing Nurhayati ${ }^{\mathrm{a}, \mathrm{b}}$, Iwan Setiawan ${ }^{\mathrm{a}}$, Gaga Irawan Nugraha ${ }^{\mathrm{d}}$, \\ Lazuardhi Dwipa ${ }^{\mathrm{e}}$, Ambrosius Purba ${ }^{\mathrm{a}}$, Unang Supratman ${ }^{\mathrm{f}}$ and Ronny Lesmana ${ }^{\mathrm{a}}$,,$*$ \\ ${ }^{a}$ Physiology Division, Department of Biomedical Sciences, Faculty of Medicine, Universitas Padjadjaran, \\ Jatinangor, Indonesia \\ ${ }^{\mathrm{b}}$ Physiology Molecular Laboratory, Biological Activity Division, Central Laboratory, Universitas Padjadjaran, \\ Jatinangor, Indonesia \\ ${ }^{\mathrm{c}}$ Graduate Program of Anti Aging and Aesthetic Medicine, Faculty of Medicine, Universitas Padjadjaran, \\ Bandung, Indonesia \\ ${ }^{\mathrm{d}}$ Biochemistry Division, Department of Biomedical Sciences, Faculty of Medicine, Universitas Padjadjaran, \\ Jatinangor, Indonesia \\ ${ }^{\mathrm{e}}$ Geriatric Subdivision, Department of Internal Medicine, Faculty of Medicine-Hasan Sadikin Hospital, \\ Universitas Padjadjaran, Bandung, Indonesia \\ ${ }^{\mathrm{f}}$ Department of Chemistry, Faculty of Mathematics and Natural Sciences, Universitas Padjadjaran, Jatinangor, \\ Indonesia
}

Received 26 November 2020

Accepted 16 December 2021

Pre-press 7 January 2022

Published 13 April 2022

\begin{abstract}
The white adipose tissue (WAT) browning process has become one of the promising methods for managing obesity. During this process, WAT is transformed into brown-like adipose tissue, which is also known as beige adipose tissue. The browning process can be activated by several inducers. One of the best candidates is peroxisome proliferator-activated receptor $\gamma(\operatorname{PPAR} \gamma$ ) agonist. Nutmeg (Myristica fragrans Houtt) is a natural PPAR $\alpha / \gamma$ partial agonist that is known to contribute to the browning effect. This study aimed to explore the potential effect of nutmeg seed extract (NuSE) on body weight reduction and uncoupling protein (UCP)1, UCP2, UCP3, and peroxisome proliferator-activated receptor gamma coactivator-1 PGC-1 $\alpha$ levels in aging rats. Eight male Wistar rats ( 80 weeks old) were divided into control and treatment groups. Both groups were fed a standard diet, and the treatment group was given $8.1 \mathrm{mg} / \mathrm{kg}$ body weight/day of NuSE via oral gavage for 12 weeks. After 12 weeks, the levels of UCP1, UCP2, UCP3, and PGC-1 $\alpha$ from both inguinal WAT (iWAT) and interscapular brown adipose tissue (BAT) were examined. We observed that the administration of NuSE has no significant effect to the decreasement of rats body weights $(p=0.464)$, levels of UCP1 $(p=0.686)$, UCP2 $(p=0.360)$, UCP3 $(p=0.076)$, and PGC-1 $\alpha(p=0.200)$.
\end{abstract}

Keywords: Adipose tissue, browning, nutmeg, aging

\section{Introduction}

${ }^{*}$ Corresponding author: Ronny Lesmana, Physiology Division, Department of Biomedical Sciences, Faculty of Medicine, Universitas Padjadjaran, Jalan Raya Jatinangor Km 21.6 Bandung, Indonesia. Tel.: +62 22 779458; E-mail: ronny@unpad.ac.id.

A fundamental process associated with aging is dysregulation of the energy homeostasis, and many age-related genes belong to evolutionarily conserved pathways that also control energy metabolism, with the adipose organ as an important metabolic player

ISSN 2451-9480 @ 2021 - The authors. Published by IOS Press. This is an Open Access article distributed under the terms of the Creative Commons Attribution-NonCommercial License (CC BY-NC 4.0). 
in the regulation of whole-body energy homeostasis [1]. The main parenchymal cells of the adipose organ are adipocytes, and so far two cell types of opposing functions have been reported: adipocytes in the white adipose tissue (WAT) usually store energy surplus in the form of lipids in two compartments, the subcutaneous (sWAT) and the visceral (vWAT) white adipose depot, and are characterized by a large unilocular lipid droplet and a low density of mitochondria, whereas adipocytes in the brown adipose tissue (BAT) can massively dissipate energy via non shivering thermogenesis and are characterized by multilocular small lipid droplets and a high abundance of mitochondria [2]. Several mechanisms are shown to be associated with BAT decline with aging, including loss of mitochondrial function, impairment within the sympathetic system and age-induced alterations in endocrine signals and inflammation. During aging, there's a big decline in UCP1 activity, a protein uniquely expressed within the inner mitochondrial membrane of brown adipocytes. In particular, mitochondrial dysfunction with aging is characterized by a rise in mitochondrial DNA mutations and a discount in biogenesis and biological process [3-5].

Recently, the white adipose tissue (WAT) browning process has become one of the promising methods for treating obesity. During the browning process, WAT can be transformed into a brown-like adipose tissue (also known as a beige adipose tissue) after a certain stimulation [6, 7]. Activated beige adipose tissue have exert thermogenic effect which is percent contribution of beige fat to total energy expenditure (7\%) was estimated to be comparable to BAT $(6.3 \%)$, in an individual with $100 \mathrm{~g}$ of BAT and $15 \mathrm{~kg}$ of WAT.

As it was reported that adults only have a limited number of brown adipose tissue (BAT) [8], the thermogenic effect exerted by beige adipose tissue is considered to have more significant metabolic effect. There are several well-researched inducers for this process, including $\beta 3$-adrenergic receptor ( $\beta 3 \mathrm{AR})$ agonist [9], cold [10], peroxisome proliferator-activated receptor $\gamma$ (PPAR $\gamma)$ agonist [11-13], fibroblast growth factor 21 (FGF21) [14], irisin [15], and exercise [16-18]. Aside from the inducers aforementioned, several nutraceuticals have also been proven to stimulate the browning process through various mechanisms. Several nutraceuticals that have been demonstrated to exert a browning effect include resveratrol, quercetin, green tea, curcumin, and berberine [14]. As some natural agonists of $\beta 3 A R$ have been reported to induce the browning process, the natural agonist of PPAR $\gamma$ has recently been considered to have the same potency $[15,16]$. Among several natural agonists of PPAR $\gamma$, nutmeg (Myristica fragrans Houtt) is hypothesized to exert a browning effect. Several mechanisms are shown to be associated with BAT decline with aging, including loss of mitochondrial function, impairment within the sympathetic system and age-induced alterations in endocrine signals and inflammation. During aging, there's a big decline in UCP1 activity, a protein uniquely expressed within the inner mitochondrial membrane of brown adipocytes. In particular, mitochondrial dysfunction with aging is characterized by a rise in mitochondrial DNA mutations and a discount in biogenesis and biological process. For this reason, aging could also be related to an impairment in brown adipogenicity stem/progenitor cell function and consequently with a discount within the regenerative potential of BAT with storage of dysfunctional brown adipocytes. One of the potential PPAR $\gamma$ agonists, Rosiglitazone, reportedly induces browning of WAT and increases mitochondrial biogenesis. However, rosiglitazone as long term treatment are still debatable for ageing due to its possible side effect [12]. Therefore, study using natural nutraceutical as PPAR $\gamma$ agonist such as nutmeg need to do.

Nutmeg, a native herb from the Maluku Island, Indonesia, and Grenada Island, has been used as herb and traditional medicine for many centuries [17]. It has been proven to have numerous beneficial effects on health, including anti-inflammatory and analgesic [18], anticonvulsant [19], antibacterial and antifungal $[20,21]$, antidiabetic and antidyslipidemia [22, 23], as well as antioxidant effects [24, 25]. In 2012, Lestari et al. reported that nutmeg has partial agonism effects of both PPAR $\alpha$ and PPAR $\gamma$ [22]. Moreover, it has been proven to induce mitochondrial biogenesis of rats through the increase in the $P G C 1-1 \alpha$ expression level [26]. Numerous transcription factors and pathways are involved in the browning process, including PGC- $1 \alpha$, PPAR $\gamma$, and PR domain containing 16 (PRDM16) [27].

Our previous study demonstrated that nutmeg seed extract (NuSE) can induce macroscopic and microscopic morphological changes of iWAT, significantly increase the uncoupling protein (UCP) 3 and PGC$1 \alpha$ levels, and offer the tendency to increase the UCP1 and UCP2 levels in young rats. Although these results indicate that nutmeg potentially alters the WAT browning process in young rats, its effect on UCPs and the WAT browning process in aging rats still remains unclear. During the aging process, several changes occur, including those that affect 
the physiological functions of the body (e.g., mitochondrial function) [28]. Ageing may be considered as cumulative impaired tissue homeostasis. Ageing likely involves alteration of several connected biological process involving redox state, metabolism, and synthesis-degradation of macromolecules. Several studies showed that there are possible crosslink between mitochondrial dysfunction associated with increased reactive oxygen species (ROS) production. Dysfunctional mitochondria predispose to altered lipid metabolism and may also influenced the quantity and distribution of adipose tissue (i.e., increase in adiposity and decline in BAT's function and mass) [29]. In this study, we aimed to observe the effects of NuSE as one of the natural nutraceutical with PPAR $\gamma$ agonist activity on several proteins involved in the WAT browning process (i.e., UCP1 and PGC- $1 \alpha$ ) and other UCPs (i.e., UCP2 and UCP3) in aging rats.

\section{Materials and methods}

\subsection{Nutmeg extract (NuSE)}

In this study, we used NuSE that has been purified from safrole and myristicin owing to their hallucinogenic and hepatotoxicity effects. The protocols of extraction and purification have been adopted from our previous study [30]. The nutmeg extract used in this study is free safrole and myristicin nutmeg extract. The dried seeds of Myristica fragrance were collected during dry season from Maluku and West Java Island. Thirty kilograms of powdered seed was extracted with 2251 ethanol $95 \%$ at room temperature using a pilot scale extractor with a circulator rate of 150-200 rpm for 30 minutes. The extract was evaporated at a temperature range of $40-60^{\circ} \mathrm{C}$ and pressure range of 400-500 $\mathrm{mmHg}$ [30].

\subsection{Ethical statement}

The study protocol was reviewed and approved by the Animal Ethics Committee of Universitas Padjadjaran, Bandung, Indonesia, and all animal procedures were performed according to its guidelines (ethics approval no. 1080/UN6.KEP/EC/2019).

\subsection{Animal model}

Eighty-week-old male Wistar rats $(n=8)$ with body weights within the range from 400 gram to 520 gram provided by PT Bio Farma, Bandung, Indonesia, were used in this study. After adaptation for 1 week, the rats were randomly divided into two groups: control and NuSE groups. The rats in the control group were given $2 \%$ pulvis gummi arabicum, whereas the rats in the NuSE group were given $8.1 \mathrm{mg} / \mathrm{kg} \mathrm{BW} /$ day NuSE via oral gavage for 12 weeks [30]. The specific dose was adjusted according to individual body weight. Both groups were fed a standard diet (containing 20\% protein and $4 \%$ fat) and water ad libitum and were kept under the following conditions: temperature of $24^{\circ} \mathrm{C}$, relative humidity of $55 \%$, and 12-h light-dark cycle. The body weights of each rat and food consumption were recorded on a weekly basis using analytic scales. Food consumption (in grams) was calculated as leftover. After 12 weeks, the animals were sacrificed using a carbon dioxide anaesthesia, and the adipose tissue was then dissected and stored at $-80^{\circ} \mathrm{C}$ for further analysis. In this study, we used subcutaneous (inguinal) WAT and interscapular BAT from both group (WAT from control group as negative control, BAT from control group as positive control and WAT from NuSE group as treatment sample).

\subsection{Western blot analysis}

In this study, the protocols of western blot procedures were adopted from our previous study. The dissected samples was homogenised in lysis buffer containing $10 \mathrm{mM}$ Tris- $\mathrm{HCl}$ (pH 7.8), $150 \mathrm{mM}$ $\mathrm{NaCl}, 1 \mathrm{mM}$ EDTA, $1 \%$ Nonidet P-40, and protease inhibitors. After centrifugation, protein samples were heat denatured at $96^{\circ} \mathrm{C}$ for 5 minutes. Samples $(10 \mu \mathrm{g} /$ lane $)$ were separated by SDS-PAGE and were then transferred to a nitrocellulose membrane (GE Healthcare) for 1 hour at room temperature and blocked overnight at $4{ }^{\circ} \mathrm{C}$ in $2 \%$ blocking reagent (GE Healthcare) in Tris-buffered saline buffer with $0.1 \%$ Tween 20 . Immunoblotting was performed using this following : rabbit monoclonal UCP1 (\#U6382) and UCP3 (\#U7757) from SigmaAldrich, UCP2 (\#89326) from Cell Signalling, and mouse monoclonal PGC-1 $\alpha$ (PA5-38022) and $\beta$ actin (MA5-15739) from Thermo Fisher Scientific with a 1:1000 dilution with phosphate-buffered saline with Tween-20 (PBST). Secondary antibody antimouse (C90130-03) and anti-rabbit (C81106-06) from RND were used with a 1:15000 dilution with PBST. The LI-COR software was used to analyse the band intensities. 

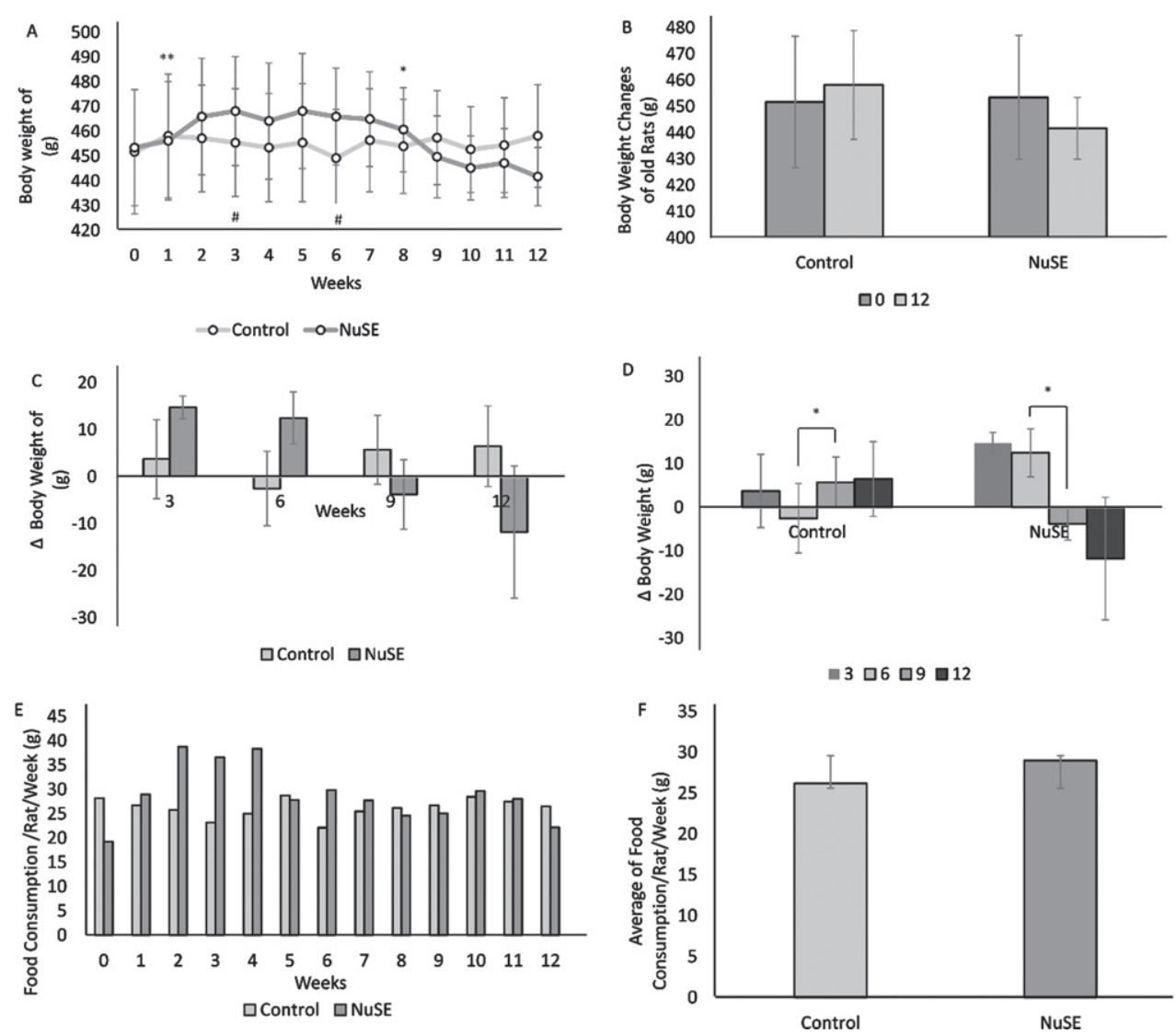

Fig. 1. Although body weight demonstrated a decreasing trend in the NuSE group at week 9 until week 12 (A), no significant difference was observed between both groups at the end of the study (B) and at weeks 3, 6, 9, and 12 (C). Interestingly, significant body weight changes were found at week 6 to week 9 in both groups (D). No significant difference in weekly food consumption between both groups was observed (E, F).

\subsection{Statistical analysis}

Statistical analysis was conducted using the SPSS software version 25. Homogeneous data were analyzed via one-way analysis of variance. Statistical significance was designated at $p<0.05$.

\section{Results}

\subsection{NuSE has a tendency to reduce body weight but does not alter food consumption}

After 12 weeks, the average body weight in the control group demonstrated an increasing trend $(p=0.503)$, whereas that in the NuSE group exhibited a decreasing trend $(p=0.464)$ (Figs. 1A and 1B). Although we did not find any significant difference in the body weight changes between the control and NuSE groups (Fig. 1 C), the body weight changes in both groups showed a significant difference from week 6 to week 9 (Fig. 1D). Moreover, no significant difference was observed in food consumption between the control and NuSE groups (Figs. 1E and $1 \mathrm{~F}$ ).

\subsection{NUSE has a tendency to increase the UCPI, $U C P 2, U C P 3$, and PGC-1 $\alpha$ levels}

We observed that NuSE has no significant effect to the increasement levels of UCP1 $(p=0.686)$, UCP2 $(p=0.360)$, UCP3 $(p=0.076)$, and PGC$1 \alpha(p=0.200)$ in inguinal WAT (iWAT) (Figs. 2A and $2 \mathrm{~B})$.

\section{Discussion}

We observed that the average body weight of rats in the NuSE group exhibited an increasing trend, 


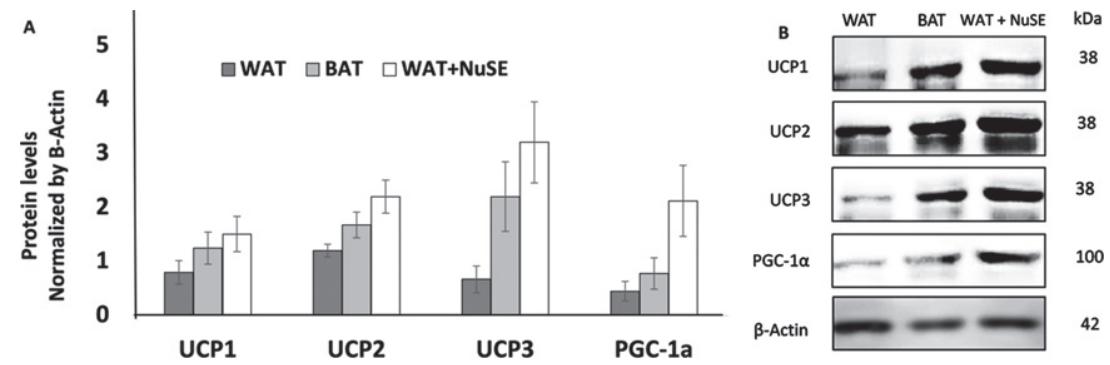

Fig. 2. (A) NuSE has a tendency to increase the levels of UCP1, UCP2, UCP3, and PGC-1 $\alpha(n=4)$; (B) representative western blots showed an increase in UCP1, UCP2, UCP3, and PGC-1 $\alpha$ levels in the NuSE group.

whereas that of rats in the control group exhibited a decreasing tend (Fig. 1A). However, after 12 weeks of $\mathrm{NuSE}$ administration, the average final body weights in both groups demonstrated no significance difference (Fig. 1B). These results are slightly different from those of our previous study on young rats. In our previous study, we observed that the administration of NuSE tended to increase the average body weights of both groups. However, the increase in the control group was higher. The aging process was one of the several factors contributing to the body composition and fat mass changes. Even though the body weight and total body fat of both rodents and human tended to increase with age [31], the muscle mass, classical BAT, and beige adipose tissue tended to decrease during aging [32]. Aging also caused a decline in lipid metabolism and fatty acid oxidation, especially in respiring tissue (i.e., skeletal muscle) [33]. These differences could possibly induce the different effect of NuSE on body weight in young and aging rats. Although nutmeg has been reported as a potent appetite enhancer [27], this study demonstrated that it did not alter food consumption in aging rats. Thus, the mechanism of nutmeg in body weight remains unclear and needs further investigation.

As nutmeg has been reported to exhibit potency as dual agonism of PPAR $\alpha / \gamma$ [25], it is considered to exhibit potency in inducing the WAT browning process. Our previous study reported that nutmeg may indeed affect the WAT browning process in young rats, as morphological changes in WAT (both macroscopic and microscopic) and increases in PGC- $1 \alpha$ and UCP1 levels were observed [34]. In line with our previous study, an increase in the UCP1 levels in aging rats was also observed; however, the increase was not statistically significant (Fig. 2A). We also found that the administration of NuSE has a tendency to increase the PGC- $1 \alpha$ levels (Fig. 2A). Several stud- ies reported that chronic administration of PPARy and PPAR $\alpha$ agonist can induce $P G C-1 \alpha$ expression [11] In addition, Veronica et al. reported that NuSE can induce mitochondrial biogenesis in the brain of young rats, indicated by the upregulation of $P G C$ $1 \alpha$ [28]. There also another interesting finding that PPAR $\gamma$ ligand rosiglitazone able to improve glucose metabolism and mitochondrial functionality in 14 months-old mice. Aged mice with Rosiglitazone treatment showed better insulin sensitivity, glucose uptake in adipocyte, increased browning in iWAT, increases oxidative number and mitochondria in muscle [35]. The insignificant results in this study are possibly caused by nutmeg's partial PPAR $\gamma$ agonism. Several studies reported different results of the induction of partial PPAR $\gamma$ agonist in the WAT browning process. Ohno et al. suggested that the WAT browning process requires chronic stimulation with full PPAR $\gamma$ agonist, whereas partial PPAR $\gamma$ agonist had minimal or no effect on the browning process [17]. Another study also reported that although the administration of weak PPAR $\gamma$ agonist can significantly increase the $U c p l$ expression level, its effect on the browning process still remains unclear. Meanwhile, Coelho et al. reported different results, in which partial PPAR $\gamma$ agonist can induce thermogenesis-related genes in visceral WAT, including $U C P 1$ expression [13].

During the aging process, a reduction of both the function and mass of classical BAT (marked by the decrease in UCP1) and beige adipose tissue (marked by the decrease in CD137/TMEM26+) was observed. The decrease in beige adipose tissue during aging could be due to the decline in WAT's ability to differentiate and SIRT1 level [36]. A previous study reported that SIRT1 plays a significant role in the browning process by promoting deacetylation of PPAR $\gamma$ and facilitating the recruitment and docking of PRDM16 [37]. The decrease in beige adipose tissue during the aging process could be attributed to 
the insignificant increase in UCP1 and PGC-1 $\alpha$ in this study.

The effect of NuSE on the UCP2 and UCP3 levels has not yet been established. We observed that a 12-week administration of NuSE can increase both the UCP2 and UCP3 levels in iWAT of aging rats; however, both increases were insignificant. Several studies reported similar results. Fukui et al. reported that induction with weak PPAR $\gamma$ agonist can increase the UCP2 expression; however, the increase is insignificant [38]. Another research also observed that induction with PPAR $\alpha$ agonist tends to increase the UCP2 expression [31]. However, it remains unclear whether induction with full PPAR $\alpha$ or PPAR $\gamma$ agonist can significantly increase $U C P 2$. The gene expression of UCP3 in WAT has been reported to increase the WAT browning process induced by thyroid hormones, $\beta 3 \mathrm{AR}$, and leptin [32]. However, whether UCP3 was involved in the browning process remains unclear. A study in 2015 reported that PPAR $\gamma$ and PGC- $1 \alpha$ can upregulate the UCP3 expression in the skeletal muscle [40]. The insignificant increases in UCP2 and UCP3 in this study might also be affected by the decline in the muscle mass and metabolism of fatty acid during aging [31, 33].

Our previous study in young rats reported an insignificant increase in UCP2 and a very significant increase in UCP3 protein in iWAT after induction with partial PPAR $\alpha / \gamma$ agonist. Although both the UCP2 and UCP3 levels demonstrated tendencies to increase in this study (Fig. 2A), we suspected that the aging process contributes to the different results. A study in 2015 reported that the expression of UCP 2 in rats varies according to the age and organs [33]. The expression and levels of UCP 2 in the liver were higher in older rats. Meanwhile the expression of $U C P 2$ in the skeletal muscle was higher in younger rats. The expression of UCP3 in the skeletal muscle was also found to be higher in older rats [33]. However, the roles of these UCPs in the WAT browning process remain unclear and need further investigation.

\section{Conclusion}

Taken together, twelve week administration of NuSE might reduce the body weight of aging rats, and this effect might not be induced via reduction of food consumption. Additionally, eventhough, NuSE show a tendency to increase the levels of UCP1, $\mathrm{UCP} 2$, UCP3, and PGC- $1 \alpha$ in the iWAT of aging rats. Interestingly, the UCP2 and UCP3 levels exhibited tendencies to increase, the role of these UCPs in the browning process remains unclear. These results indicate that NuSE may potentially be involved in the WAT browning process of aging rats. Nonetheless, further investigation is still needed.

\section{Acknowledgments}

The authors have no acknowledgments.

\section{Funding}

The authors report no funding.

\section{Conflict of interest}

The authors have no conflict of interest to report.

\section{References}

[1] Curtis R, Geesaman BJ, DiStefano PS. Ageing and metabolism: drug discovery opportunities. Nat Rev Drug Discov. 2005;4:569-80.

[2] Cinti S. The adipose organ at a glance. Dis Model Mech. 2012;5:588-94.

[3] Zoico E, Rubele S, De Caro A, et al. Brown and Beige Adipose Tissue and Aging. Front Endocrinol (Lausanne). 2019;10:368

[4] Lenaz G. Role of mitochondria in oxidative stress and ageing. Biochim Biophys Acta Bioenerget. 1998;1366:53-67. 10.1016/S0005-2728(98)00120-0

[5] Poher A-L, Altirriba J, Veyrat-Durebex C, RohnerJeanrenaud F. Brown adipose tissue activity as a target for the treatment of obesity/insulin resistance. Front Physiol. 2015;6:4. 10.3389/fphys.2015.00004

[6] Wu J, Cohen P, Spiegelman BM. Adaptive thermogenesis in adipocytes: is beige the new brown? Genes Dev. 2013;27(3):234-50.

[7] Giralt M, Villarroya F. White, brown, beige/brite: different adipose cells for different functions? Endocrinology. 2013;154(9):2992-3000.

[8] van Marken Lichtenbelt W, Vanhommerig J, Smulders N, Drossaerts J, Kemerink G, Bouvy N, et al. Cold-activated brown adipose tissue in healthy men. $\mathrm{N}$ Engl $\mathrm{J}$ Med. 2009;360(15):1500-8.

[9] Richard JE, López-Ferreras L, Chanclón B, Eerola K, Micallef P, Skibicka KP, et al. CNS $\beta 3$-adrenergic receptor activation regulates feeding behavior, white fat browning, and body weight. Am J Physiol Endocrinol Metab. 2017;313(3):E344-58

[10] Ravussin Y, Xiao C, Gavrilova O, Reitman ML. Effect of intermittent cold exposure on brown fat activation, obesity, and energy homeostasis in mice. PLoS One. 2014;9(1):1-9. 
[11] Petrovic N, Walden TB, Shabalina IG, Timmons JA, Cannon B, Nedergaard J. Chronic peroxisome proliferator-activated receptor gamma (PPAR gamma) activation of epididymally derived white adipocyte cultures reveals a population of thermogenically competent, UCP1-containing adipocytes molecularly distinct from classic brown adipocy. J Biol Chem. 2010;285(10):7153-64.

[12] Ohno H, Shinoda K, Spiegelman BM, Kajimura S. PPAR gamma agonists induce a white-to-brown fat conversion through stabilization of PRDM16 protein. Cell Metab. 2012;15(3):395-404.

[13] Coelho MS, De Lima CL, Royer C, Silva JB, Oliveira FCB, Christ CG, et al. GQ-16, a TZD-derived partial PPAR $\gamma$ agonist, induces the expression of thermogenesis-related genes in brown fat and visceral white fat and decreases visceral adiposity in obese and hyperglycemic mice. PLoS One. 2016;11(5):1-20.

[14] Fisher M, Kleiner S, Douris N, Fox EC, Mepani RJ, Verdeguer F, et al. FGF21 regulates PGC-1 alpha and browning of white adipose tissues in adaptive thermogenesis. Genes Dev. 2012;26(3):271-81.

[15] Bostrom P, Wu J, Jedrychowski MP, Korde A, Ye L, Lo JC, et al. A PGC1- a -dependent myokine that drives brown-fatlike development of white fat and thermogenesis. Nature. 2012;481(7382):463-8.

[16] Preppler W, Townsend I, Knuth C, Foster M, Wright D. Subcutaneous inguinal white adipose tissue is responsive to, but dispensable for, the metabolic health benefits of exercise. Am J Physiol Endocrinol Metab. 2018;314(1):E66-77.

[17] Flouris AD, Dinas PC, Valente A, Andrade CMB, Kawashita $\mathrm{NH}$, Sakellariou P. Exercise-induced effects on UCP1 expression in classical brown adipose tissue: a systematic review. Horm Mol Biol Clin Invest. 2017;31(2):1-13.

[18] Motiani P, Virtanen KA, Motiani KK, Eskelinen JJ, Middelbeek RJ, Goodyear LJ. Decreased insulin-stimulated brown adipose tissue glucose uptake after short-term exercise training in healthy middle-aged men. Diabetes Obes Metab. 2017;19(10):1379-88.

[19] Abourashed EA, El-alfy AT. Chemical diversity and pharmacological significance of the secondary metabolites of nutmeg (Myristica fragrans Houtt.). Phytochem Rev. 2016;15(6):1035-56.

[20] Asgarpanah J, Kazemivash N. Phytochemistry and pharmacologic properties of Myristica fragrans Houtt.: a review. Afr J Biotechnol. 2012;11(65):12787-93.

[21] Wahab A, Haq R, Ahmed A, Khan R, Raza M. Anticonvulsant activities of nutmeg oil of Myristica fragrans. Phytother Res. 2009;23:153-8.

[22] Ibrahim T, Opawale B, Oyinloye J. Antibacterial activity of herbal extracts against multi drug resistant strains of bacteria from clinical origin. Life Sci Leafl. 2011;15:490-8.

[23] Valente V, Jham G, Dhingra O, Ghiviriga I. Compositition and antifungal activity of the Brazilian Myristica fragrans Houtt essential oil. J Food Saf. 2011;31(2):197-202.

[24] Lestari K, Hwang J, Kariadi SH, Wijaya A, Ahmad T, Subarnas A, et al. Screening for PPAR gamma agonist from Myristica fragrans Houtt seeds for the treatment of type 2 diabetes by in vitro and in vivo. Med Heal Sci Journal, MHSJ. 2012;12:7-15.

[25] Lestari K, Diantini A, Barliana MI, Achmad TH, Subarnas A, Abdulah R, et al. Potential natural dual agonist PPAR $\alpha / \gamma$-induced antidiabetic and anti-dyslipidemic properties of safrole-free nutmeg seed (Myristica fragrans Houtt) extract. J Nat Prod. 2019;9:248-53.

[26] Gupta AD, Bansal VK, Babu V, Maithil N. Chemistry, antioxidant and antimicrobial potential of nutmeg (Myristica fragrans Houtt). J Genet Eng Biotechnol. 2013;11(1):25-31.

[27] Wibowo DP, Febriana Y, Riasari H, Auilifa DL. Essential oil composition, antioxidant and antibacterial activities of nutmeg (Myristica fragrans Houtt) from Garut West Java. IJPST. 2018;5(3):82-7.

[28] Veronica F, Lubis L, Arifin S, Fitri LL, Rizal A, Ambrosius $\mathrm{P}$, et al. A preliminary study of the effect of PPAR- $\gamma$ agonist from Myristica fragrans houtt seed extract on the biogenesis of rat infant's brain mitochondria and D1 dopamine receptor. Bali Med J. 2018;7(3):574-7.

[29] Lo KA, Sun L. Turning WAT into BAT: a review on regulators controlling the browning of white adipocytes. Biosci Rep. 2013;33(5):711-9.

[30] Pratiwi YS, Lesmana R, Goenawan H, Sylviana N, Setiawan I, Tarawan VM, et al. Nutmeg extract increases skeletal muscle mass in aging rats partly via IGF1-AKT-mTOR pathway and inhibition of autophagy. Evid Based Complement Altern Med. 2018;2018(2810840):1-9.

[31] Nakatani T, Tsuboyama-Kasaoka N, Takahashi M, Miura S, Ezaki O. Mechanism for peroxisome proliferator activated receptor-alpha activator induced up-regulation of UCP2 mRNA in rodent hepatocytes. J Biol Chem. 2002;277: 9562-9.

[32] Gong D, He Y, Karas M, Reitman M. Uncoupling Protein-3 Is a Mediator of Thermogenesis Regulated by Thyroid Hormone, beta 3 adrenergic agonist, and leptin. J Biol Chem. 1997;272(39):24129-32.

[33] Willoughby DS. Factors Regulating Uncoupling Protein-3 (UCP-3) Expression in Skeletal Muscle. J Nutr Heal Food Eng. 2015;3(1):285-91.

[34] Lesmana R, Siannoto M, Nugraha GI, et al. Nutmeg extract potentially alters characteristics of white adipose tissue in rats. Vet Med Sci. 2021;7:512-20. https://doi.org/10.1002/vms3.383

[35] Xu L, Ma X, Verma N, Perie L, Pendse J, Shamloo S, Marie Josephson A, Wang D, Qiu J, Guo M, Ping X, Allen M, Noguchi A, Springer D, Shen F, Liu C, Zhang S, Li L, Li J, Xiao J, Lu J, Du Z, Luo J, Aleman JO, Leucht P, Mueller E. PPAR $\gamma$ agonists delay age-associated metabolic disease and extend longevity. Aging Cell. 2020;19:e13267. https://doi.org/10.1111/acel.13267

[36] Tamiris A, Rachid L, Penna-de-carvalho A, Bringhenti I, Aguila MB, Mandarim-de-lacerda CA, et al. Fenofibrate (PPAR alpha agonist) induces beige cell formation in subcutaneous white adipose tissue from diet-induced male obese mice. Mol Cell Endocrinol. 2015;402:86-94.

[37] Hondares E, Rosell M, Díaz-delfín J, Olmos Y, Monsalve M, Iglesias R, et al. Peroxisome proliferator-activated receptor alpha (PPAR alpha) induces PPAR gamma coactivator 1 alpha (PGC-1 alpha) gene expression and contributes to thermogenic activation of brown fat: involvement of PRDM16. J Biol Chem. 2011;286(50):43112-22.

[38] Fukui Y, Masui S, Osada S, Umesono K, Motojima K. A new thiazolidinedione, NC-2100, which is a weak PPAR-gamma activator, exhibits potent antidiabetic effects and induces uncoupling protein 1 in white adipose tissue of KKAy obese mice. Diabetes. 2000;49(5):759-67. 